\title{
EXPERIMENTAL CHARACTERIZATION OF ULTRAVIOLET RADIATION OF AIR IN A HIGH ENTHALPY PLASMA TORCH FACILITY
}

\author{
C. J. Casses ${ }^{1}$, P. J. Bertrand ${ }^{1}$, C. M. Jacobs ${ }^{1,2}$, \\ M. E. Mac Donald ${ }^{1,2}$, and Ch. O. Laux ${ }^{1,2}$ \\ ${ }^{1}$ Ecole Centrale, Paris \\ Châtenay-Malabry 92290, France \\ ${ }^{2}$ Laboratoire EM2C \\ CNRS UPR288, Châtenay-Malabry 92290, France
}

\begin{abstract}
During atmospheric reentry, a plasma is formed ahead of the surface of the vehicle and the excited particle present in the plasma produces radiative heating fluxes to the surface of the vehicle. A high-temperature air plasma torch operating at atmospheric pressure was used to experimentally reproduce atmospheric reentry conditions. A high-resolution and absolute intensity emission spectrum (full width at half maximum $(\mathrm{FWHM})=0.064 \mathrm{~nm})$ was obtained from 200 to $450 \mathrm{~nm}$ and then compared with computational results provided by the SPECAIR code [1]. This paper discusses the comparison of the two spectra over this wavelength range in order to confirm the validity of the calculation and provide direction to improve the calculated spectrum.
\end{abstract}

\section{INTRODUCTION}

The ultraviolet (UV) range is responsible for a large fraction of the radiation of high temperature air plasmas, through the NO $\beta, \gamma, \delta, \epsilon, \beta^{\prime}$, and $\gamma^{\prime}$ band systems, the $\mathrm{N}_{2}$ second positive system, the $\mathrm{N}_{2}^{+}$first negative system, the CN Violet bands, the $\mathrm{O}_{2}$ Schumann-Range system, and the radiation of atomic carbon. For reentry vehicles at velocities from 3 to $8 \mathrm{~km} / \mathrm{s}$, these systems represent the dominant fraction of the radiation emitted by the plasma layer located between the bow shock and the surface of the vehicle. Various spectroscopic models have been implemented in radiation codes such as SPECAIR [1] to predict the spectral intensity of these systems and generally good agreement has been obtained with measurements at medium spectral resolution (Fig. 1). However, a close inspection of the spectra shown in Fig. 1 shows a few discrepancies between the measurements and the experiments. 


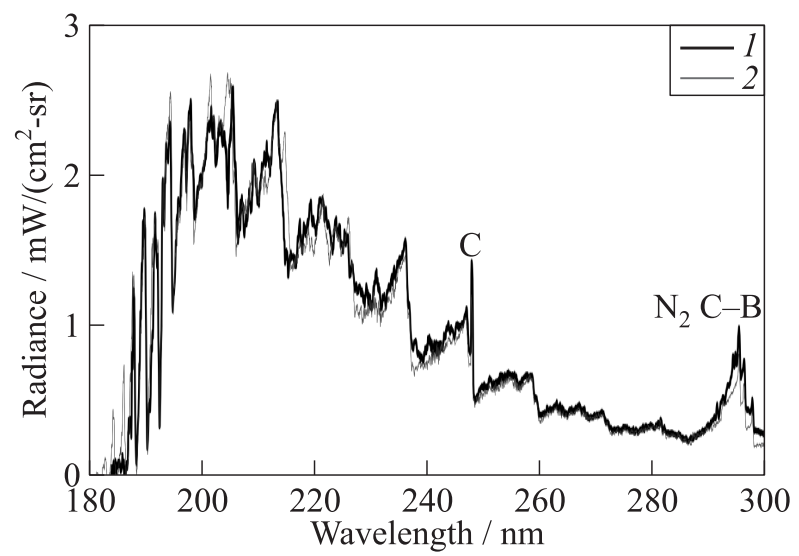

(a)

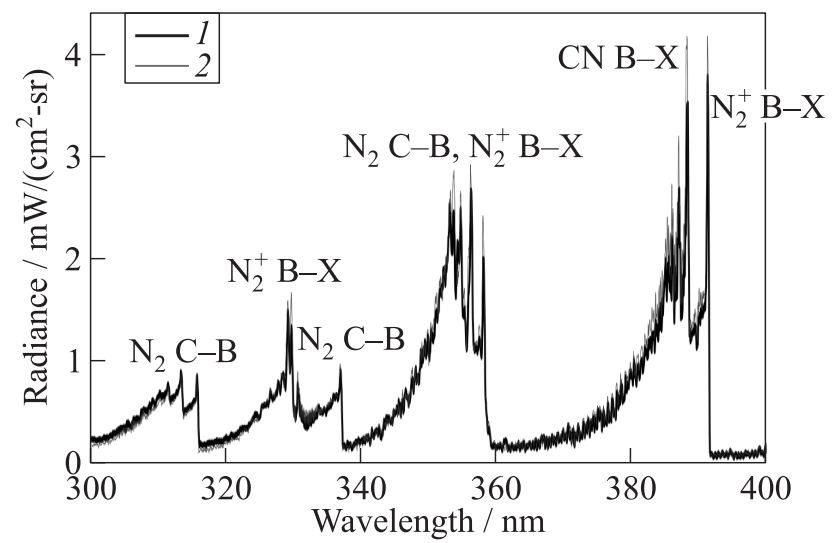

(b)

Figure 1 Comparison of measured (1) and predicted by SPECAIR (2) spectra of NO emitted by an LTE air plasma at $7500 \mathrm{~K}[2]$

The purpose of this work is to compare the predictions of the SPECAIR spectral models against measurements of the various band systems taken at a higher spectral resolution (about 5 times higher) than previously reported in [1]. To this end, a thermal air plasma with a 50-kilowatt inductively coupled plasma torch operating at atmospheric pressure has been generated. This facility provides high-enthalpy air plasma at temperatures up to $7500 \mathrm{~K}$, a range well suited for the study of all the systems emitting in the ultraviolet between 200 and $400 \mathrm{~nm}$. Because the plasma is in Local Thermodynamic Equilibrium (LTE)* , the species

${ }^{*}$ Works done by Laux [3, ch. 4] explained why we assumed chemical equilibrium with temperature profile measured from the atomic oxygen triplet around $777 \mathrm{~nm}$ and why population 
concentrations and the population distribution over the internal energy states of atoms and molecules can be modeled with the knowledge of only temperature and pressure. In this work, high-resolution measurements were made with an Acton SpectraPro 2750i spectrometer and an intensified CCD (charge coupled device) camera (PIMAX, Roper Scientific). The SPECAIR model was then compared to the measured data. Section 2 of this paper presents the experimental setup used to obtain the experimental spectra and Section 3 provides the comparison of the experimental and calculated spectra.

\section{EXPERIMENTAL SETUP}

\subsection{High Enthalpy Plasma Torch}

The facility used (Fig. 2) is a radio-frequency ( $R F$ ) inductively coupled plasma torch, TAFA Model 66, powered by an RF LEPEL Model T-50-3 power supply operating at $4 \mathrm{MHz}(120 \mathrm{kVA})$ [4]. During the torch starting phase, argon is used because its ionization potential helps initiate the high temperatures required to sustain the plasma. The argon is then slowly switched to air. The gas flow

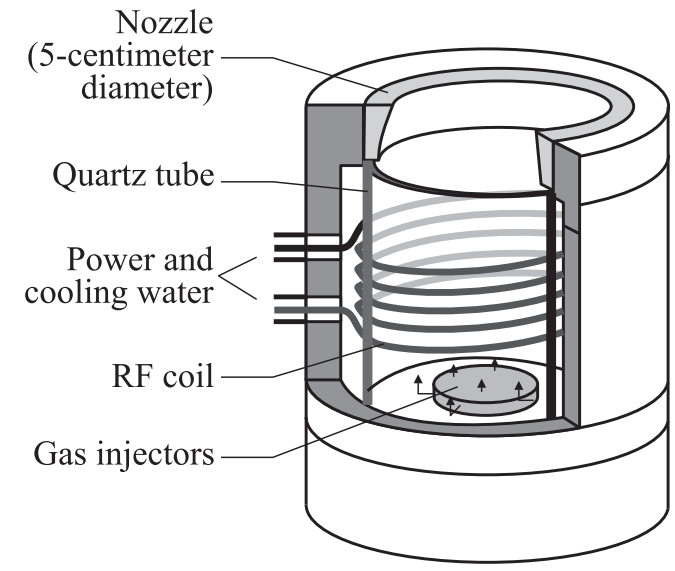

(a)

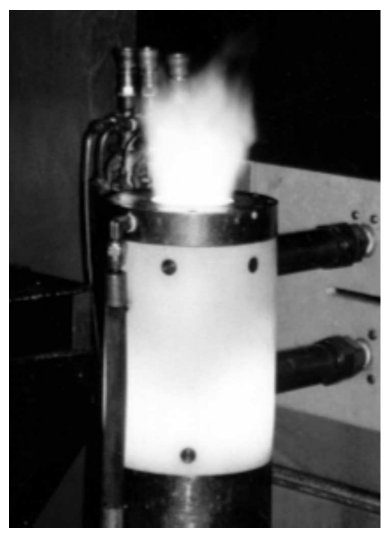

(b)

Figure 2 Torch head schematic and photograph of the air plasma plume. All measurements were performed $16 \mathrm{~mm}$ downstream of the torch exit nozzle [4]

distributions can be found using Boltzmann distributions at the LTE temperature. First, measurements of LTE temperatures from different species at $1 \mathrm{~cm}$ of the nozzle exit show that they are all superimposed together. This leads to the conclusion that the plasma near the nozzle exit where we took measurements is close to thermal equilibrium. Moreover, numerical simulations agreed with the fact that there is chemical equilibrium at the nozzle exit. 
is injected in axisymmetric fashion, with both axial and swirl injection. Swirl injection provides gas recirculation and torch stabilization. A converging copper nozzle is placed at the exit of the torch. In this study, the nozzle diameter is $5 \mathrm{~cm}$. All measurements presented in this paper were conducted $16 \mathrm{~mm}$ above the nozzle exit. At this location, the maximum velocity was approximately $20 \mathrm{~m} / \mathrm{s}$ and the measured maximum temperature was $7500 \mathrm{~K}$, corresponding to a density, $\rho$, of $3.16 \cdot 10^{-2} \mathrm{~kg} / \mathrm{m}^{3}$ and a dynamic viscosity, $\eta, 1.99 \cdot 10^{-4} \mathrm{~kg} / \mathrm{m} / \mathrm{s}[5]$. With these values, the Reynolds number at the measurement point was about 181 . The plasma jet is, therefore, laminar in the measurement region. It remains so downstream of the nozzle over a few centimetres [4]. The swirl injection, creating recirculation, stabilizes the plasma and minimizes the occurrence of temporal and local fluctuations.

\subsection{Optics Schematic}

A photograph of the optical train and spectrometer is shown in Fig. 3, and Fig. 4 presents the optical schematic. Vertical and lateral scanning capabilities are possible with a set of two off-axis parabolic and five flat mirrors on horizontal and vertical translation stages [4].
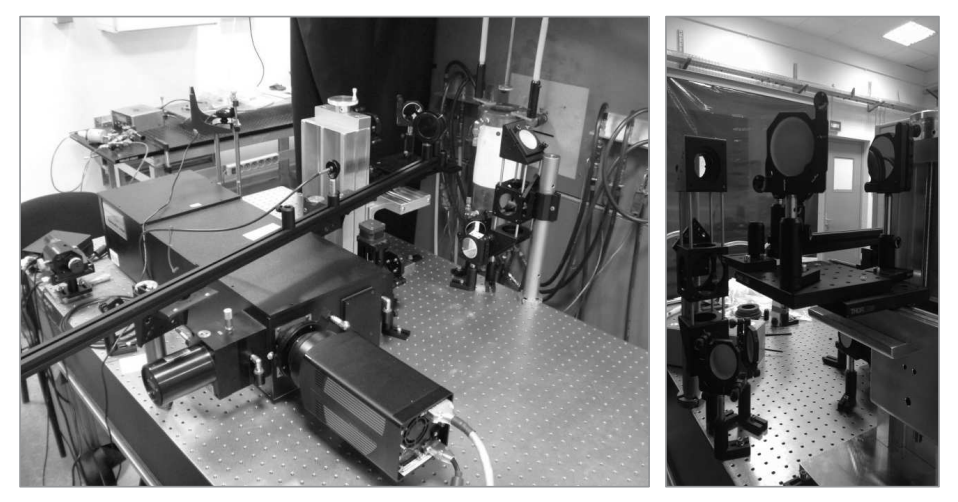

Figure 3 Optical emission spectroscopy setup and detail of mirrors

\subsection{Spectrometer}

The measurements conducted in this work used an Acton Research SpectraPro SP2750i spectrograph of focal length $750 \mathrm{~mm}$. The grating has $1200 \mathrm{~g} / \mathrm{mm}$ and is blazed at $300 \mathrm{~nm}$, providing a wavelength range of approximately $15 \mathrm{~nm}$ in one image.

The spectrometer is equipped with a vacuum UV (VUV) enhanced intensified CCD camera (Princeton Instruments PI-MAX 1024RB-UV-18mm with 


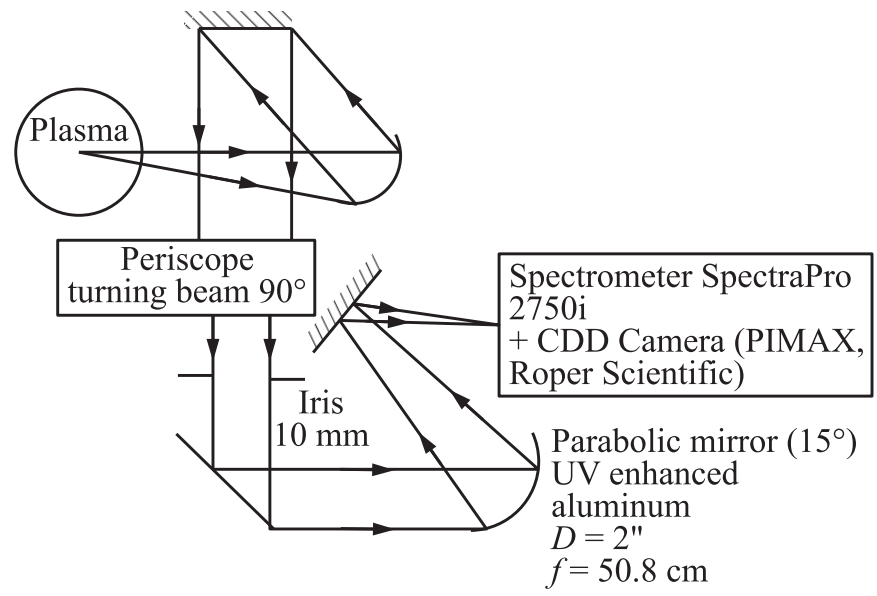

Figure 4 Schematic of the optical system with the SpectraPro spectrometer

$1024 \times 256$ pixels) allowing acquisition of emission from the VUV to the nearinfrared (140-930 nm). A HeNe laser is used to align the spectrometer with the torch, and the measurements were taken with a spectrometer entrance slit width of $25 \mu \mathrm{m}$. With this setup, the spectral resolution of the spectrometer is approximately $0.064 \mathrm{~nm}(\mathrm{FWHM})$. The spectrometer is used to measure the emission spectrum of the air plasma between 200 and $450 \mathrm{~nm}$ and to determine the radial temperature profile of the plasma using the oxygen triplet at $777 \mathrm{~nm}$.

\subsection{Calibration Lamps}

The measured spectrum was calibrated in absolute intensity using two calibration sources. An OL-550 tungsten ribbon lamp was used to calibrate the range 350$450 \mathrm{~nm}$ and an argon miniarc [4] was used from 200 to $400 \mathrm{~nm}$. The OL-550 lamp and the argon miniarc are both radiance standards with calibration traceable to NIST (National Institute of Standards and Technology) standards.

The experimental spectrum of the air plasma was calibrated in absolute intensity over the ranges $200-340$ and $340-450 \mathrm{~nm}$ by dividing the measured spectrum by the argon miniarc spectral response and by the OL-550 tungsten lamp response, respectively.

\section{RESULTS}

\subsection{Temperature Profile}

The SPECAIR calculations required the equilibrium radial temperature and the width of a slab in order to compute a spectrum. The slab is the width in 
centimeters that separate two temperature measures. Then, we added all the spectra computed with the temperature profile, a spectrum is associated with one temperature, in order to finally model the spectrum with the intensity measured at the center of the plasma.

The radial temperature of the plasma flow was determined by measuring the emission from the atomic oxygen triplet around $777 \mathrm{~nm}$. The line intensity of a specific transition is given by [3]:

$$
I\left[\frac{\mathrm{W}}{\mathrm{cm}^{3} \cdot \mathrm{sr}}\right]=\frac{n_{u}\left(\varepsilon_{u}-\varepsilon_{l}\right) A_{\mathrm{ul}}}{4 \pi} .
$$

The intensity measured along the plasma centerline is the superposition of the contributions along the plasma radius. In order to determine the radial temperature profile in the plasma, the Abel inversion technique was applied:

$$
e(r)=-\frac{1}{\pi} \int_{r}^{R} \frac{d I / d x}{\sqrt{x^{2}-r^{2}}} d x
$$

This is possible because the plasma has an axisymmetric geometry. The comparison of the intensity of both sides of the torch from the centerline confirmed this hypothesis. An inherent property of Abel inversion is that it can amplify the noise in the lateral region of the torch, which is difficult to evaluate. Some estimations based on the least-squares fitting routine are computed in $[3, \mathrm{Ap}$ pendix A].

The parameter $A_{\mathrm{ul}}, \varepsilon_{u}$, and $\varepsilon_{1}$ are tabulated [6] and for this transition are given in Table 1.

This leaves $n_{u}$ as the final unknown quantity in Eq. (1). In an LTE plasma, this quantity is related to the temperature and pressure as given in

$$
\frac{n_{u}}{g_{u}}=X_{\mathrm{O}}\left(T_{\mathrm{LTE}}\right) \frac{P}{k T_{\mathrm{LTE}}} \frac{1}{Q_{\mathrm{el}}\left(T_{\mathrm{LTE}}\right)} \exp \left(-\frac{\varepsilon_{u}}{k T_{\mathrm{LTE}}}\right)
$$

where $X_{\mathrm{O}}\left(T_{\mathrm{LTE}}\right)$ is the mole fraction of atomic oxygen in air at $P$ and $T_{\mathrm{LTE}}$, which is tabulated as a function of temperature; $P$ is the pressure at which the

Table 1 Characteristics of the atomic oxygen transition at $777 \mathrm{~nm}[6]$

\begin{tabular}{cccc}
\hline$A_{\mathrm{ul}}, \mathrm{s}^{-1}$ & $\varepsilon_{u}, \mathrm{~cm}^{-1}$ & $\varepsilon_{1}, \mathrm{~cm}^{-1}$ & $g_{u}$ \\
\hline $3.69 \cdot 10^{7}($ Uncertainty $\leq 3 \%)$ & 86629 & 73768 & 15 \\
\hline
\end{tabular}


measurement was carried out (here, $P=1 \mathrm{~atm}) ; k$ is the Boltzmann constant, and $Q_{\mathrm{el}}\left(T_{\mathrm{LTE}}\right)$ is the electronic partition function of atomic oxygen at $T_{\mathrm{LTE}}$, which for the temperatures considered here is equal to 9 . This leaves $T_{\mathrm{LTE}}$ as the only unknown, and through an iterative process, the temperature of the plasma can be determined, provided the absolute intensity, $I$, of the line is measured.

The temperature profile obtained is presented in Fig. 5.

As explained by Laux [3], by taking the logarithm of Eq. (2) and differentiating, one obtains:

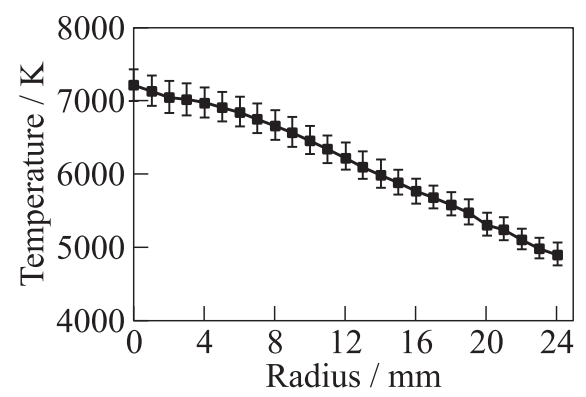

Figure 5 Radial temperature profile at $16 \mathrm{~mm}$ downstream of the plasma torch exit, with a nozzle of 5-centimeter diameter, $P=1 \mathrm{~atm}$

$$
\left|\frac{\Delta\left(n_{u} / g_{u}\right)}{n_{u} / g_{u}}\right|=\left|\frac{\Delta X_{\mathrm{O}}}{X_{\mathrm{O}}}\right|+\left|\frac{\Delta T_{\mathrm{LTE}}}{T_{\mathrm{LTE}}}\right|+\left|\frac{\Delta Q_{\mathrm{el}}}{Q_{\mathrm{el}}}\right|+\frac{\varepsilon_{u}}{k T_{\mathrm{LTE}}}\left|\frac{\Delta T_{\mathrm{LTE}}}{T_{\mathrm{LTE}}}\right| .
$$

Then, the uncertainty at an average temperature of $7000 \mathrm{~K}$, neglecting $\left|\Delta Q_{\mathrm{el}} / Q_{\mathrm{el}}\right|,\left|\Delta\left(\Delta E_{\mathrm{ul}}\right) / \Delta E_{\mathrm{ul}}\right|$, and $\left|\Delta X_{\mathrm{O}} / X_{\mathrm{O}}\right|$, which are less than $1 \%$ according to [3], is given by:

$$
\left|\frac{\Delta T_{\mathrm{LTE}}}{T_{\mathrm{LTE}}}\right|=\left(1+\frac{\varepsilon_{u}}{k T_{\mathrm{LTE}}}\right)^{-1}\left(\left|\frac{\Delta I}{I}\right|+\left|\frac{\Delta A_{\mathrm{ul}}}{A_{\mathrm{ul}}}\right|\right) .
$$

By considering the uncertainty of the spontaneous Einstein $A$ coefficient to be about $3 \%[6]$, one obtains the approximate relation at $7000 \mathrm{~K}$ :

$$
\left|\frac{\Delta T_{\mathrm{LTE}}}{T_{\mathrm{LTE}}}\right| \cong 0.05\left(\left|\frac{\Delta I}{I}\right|+0.03\right) .
$$

As the measured intensities of the oxygen line at $777.3 \mathrm{~nm}$ were obtained with accuracy better than $20 \%$, the uncertainty on the measured temperature is about $3 \%$.

Let examine the sensitivity of several features that appear in the measured spectra to the temperature. The $\mathrm{C}$ line (at $247 \mathrm{~nm}), \mathrm{N}_{2}($ at $337 \mathrm{~nm})$, and $\mathrm{N}_{2}^{+}$ (at $391 \mathrm{~nm}$ ) transitions are very sensitive to the uncertainty in temperature: an error of $\pm 3 \%$ on the temperature modifies the maximum intensity of the spectrum of these species by $60 \%, 27 \%$, and $52 \%$, respectively. The rest of the spectrum is affected by about $10 \%$ (for example, the NO transitions). Figure 6 shows the effect of a \pm 3 percent variation of the temperature on the $\mathrm{C}, \mathrm{N}_{2}, \mathrm{~N}_{2}^{+}$, and NO transitions. Because the intensity of the $\mathrm{N}_{2}^{+}$first negative spectrum at 


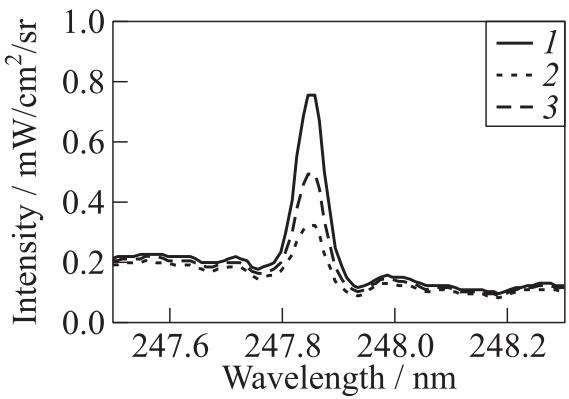

(a)

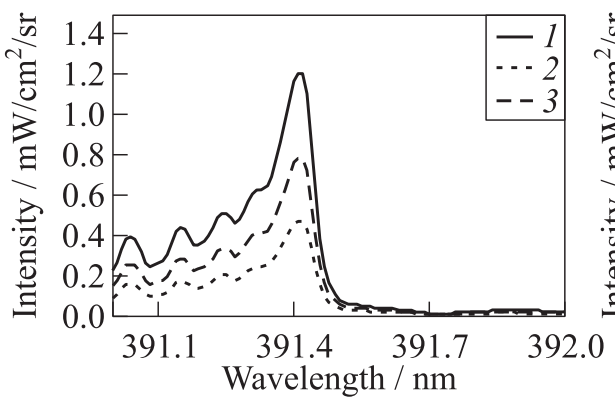

(c)

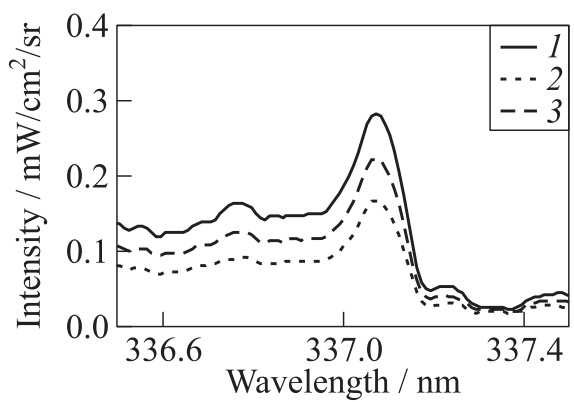

(b)

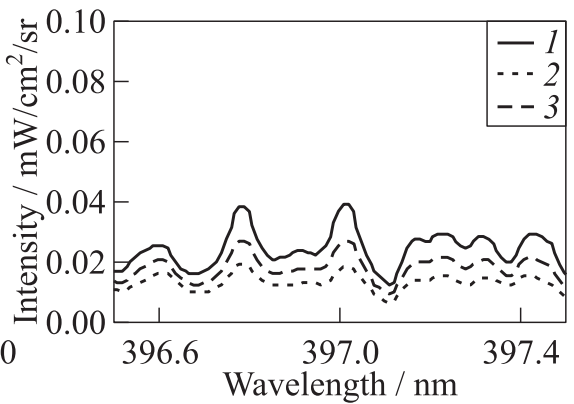

(d)

Figure 6 Variations of the $\mathrm{C}(a), \mathrm{N}_{2}(b), \mathrm{N}_{2}^{+}(c)$, and $\mathrm{NO}(d)$ transition intensities for a \pm 3 percent variation of upper (1) and lower (2) bounds of the temperature; 3 SPECAIR Spectrum. Errors $60 \%(a), 27 \%(b), 52 \%(c)$, and $10 \%(d)$

392 was better predicted by the upper bound temperature profile, all SPECAIR simulations presented in this article will be based on this upper bound temperature profile. This is further justified by the fact that self-absorption effects on the intensity of the $\mathrm{O}$ line are likely to increase the measured temperature by about 100-200 K, according to [3].

\subsection{SPECAIR Calculations}

SPECAIR is a line-by-line radiation code for computing, manipulating, and fitting spectra based on several detailed models of the transitions of air species. These models are described in several references: UV and VUV systems of NO and $\mathrm{N}_{2}$ [7], infrared systems [8], $\mathrm{N}_{2}$ and $\mathrm{N}_{2}^{+}$spectra [9,10], OH spectra [11], and $\mathrm{CN}$ and $\mathrm{C}_{2}$ spectra [12].

The plasma torch spectrum was modeled for slabs of width $1 \mathrm{~mm}$, each assumed to have homogeneous temperatures and species concentrations corre- 
sponding to those at the measured radial temperatures shown in Fig. 5. The SPECAIR calculations were made between 200 and $450 \mathrm{~nm}$, with a pressure of $1 \mathrm{~atm}$, by generating the mole fractions and considering self-absorption. The species and transitions considered for this spectral range are: C, N, O, CN violet, $\mathrm{N}_{2}(2+), \mathrm{N}_{2}^{+}$ $(1-), \mathrm{NO} \beta, \mathrm{NO} \beta^{\prime}$, NO $\delta$, NO $\epsilon$, $\mathrm{NO} \gamma, \mathrm{NO} \gamma^{\prime}$, and $\mathrm{O}_{2}$ (SchumannRunge).

The slit function was measured

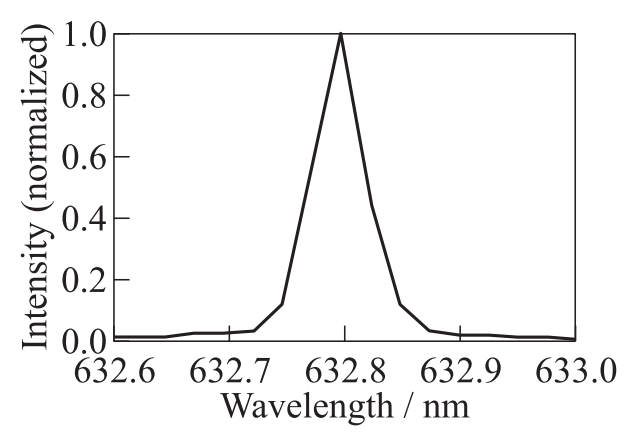

Figure 7 Spectra Pro spectrometer slit function using a $\mathrm{He}-\mathrm{Ne}$ laser at $632.8 \mathrm{~nm}$.

Figure 7 shows the slit function measured with the SpectraPro spectrometer. The equivalent slit width at half maximum is about $0.064 \mathrm{~nm}$, which is about 5 times narrower than the $0.29 \mathrm{~nm}$ FWHM of the spectra of [1] presented in Fig. 1. The slit function was convolved by SPECAIR with the predicted emission spectra so that the resulting spectra could be directly compared to the measured spectra.

\subsection{Experimental Spectra}

Spectra were obtained over two wavelength ranges: $340-450 \mathrm{~nm}$ (calibrated with the OL-550 lamp) and 200-400 nm (calibrated with the argon miniarc lamp). It can be seen in Fig. 8 that the two spectra in absolute intensity overlap with good agreement between 340 and $400 \mathrm{~nm}$, which is the range common to both radiance standards. For all results presented in this paper, the experimental spectrum calibrated with the OL 550 lamp was used for the comparison between 340 and $450 \mathrm{~nm}$ (Fig. 9) and the experimental spectrum calibrated with the argon miniarc was used for the comparison between 200 and $340 \mathrm{~nm}$ (Figs. 10 and 11). The calibration with the OL-550 is considered to be more accurate in the overlapping region than the calibration with argon miniarc.

\subsection{Comparison of Experiment and SPECAIR Calculations}

The comparison between the experiment and SPECAIR from 200 to $400 \mathrm{~nm}$ is presented in Fig. 12. The overall agreement is seen to be very good, both in terms of the shape and the absolute intensity of the various features. There are, however, some differences, which will be examined over smaller spectral range regions. 


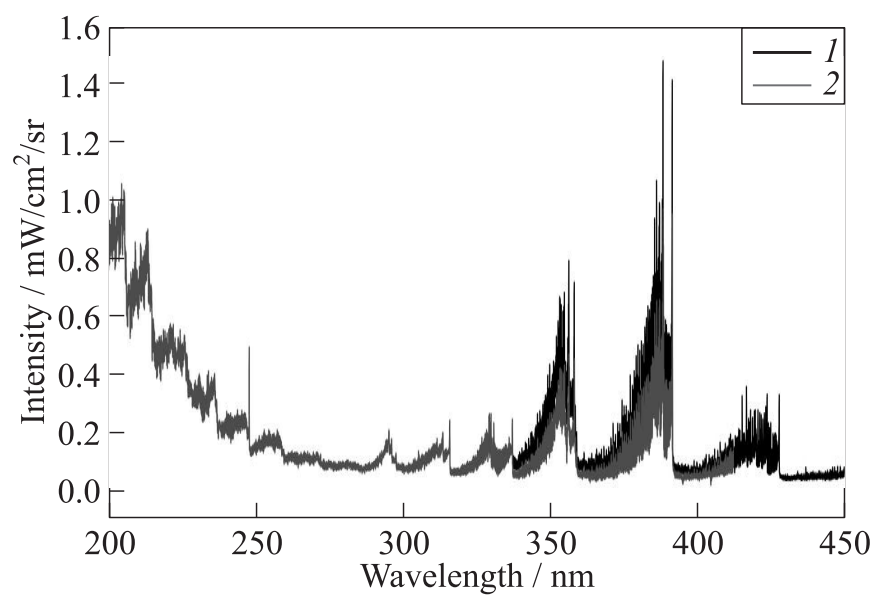

(a)

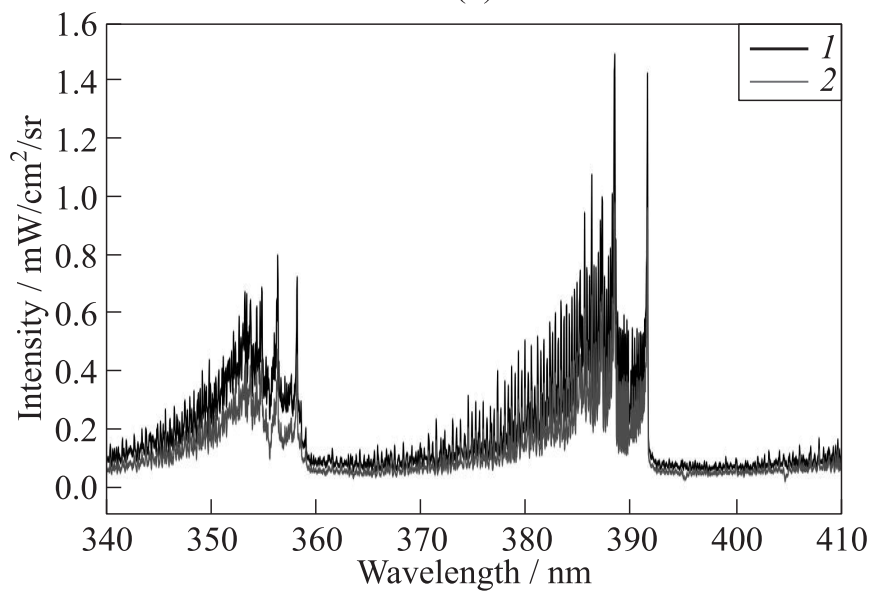

(b)

Figure 8 Absolute measured spectra calibrated with OL-550 (1) and argon miniarc (2) between 200 and $450 \mathrm{~nm}(a)$ and between 350 and $400 \mathrm{~nm}(b)$

\subsection{Species Identification}

The spectrum shown in Fig. $9 a$ contains different electronic band systems, namely, $\mathrm{N}_{2}^{+}(1-), \mathrm{N}_{2}(2+), \mathrm{O}_{2}$ Schumann-Runge, $\mathrm{NO}\left(\beta, \gamma, \delta, \epsilon, \beta^{\prime}\right.$, and $\left.\gamma^{\prime}\right)$, and $\mathrm{CN}$ violet, as well as one atomic line of carbon. At the considered temperatures $(7400 \mathrm{~K}), \mathrm{NO} \beta$, NO $\delta$, NO $\epsilon$, and $\mathrm{NO} \gamma$ emit from 200 to $280 \mathrm{~nm}$, and $\mathrm{N}_{2}^{+}, \mathrm{CN}$, and $\mathrm{N}_{2}$ are responsible for most of the radiation between 280 and $400 \mathrm{~nm}$. The atomic line of carbon is at $248 \mathrm{~nm}$. Let now consider smaller ranges for a more detailed comparison. 


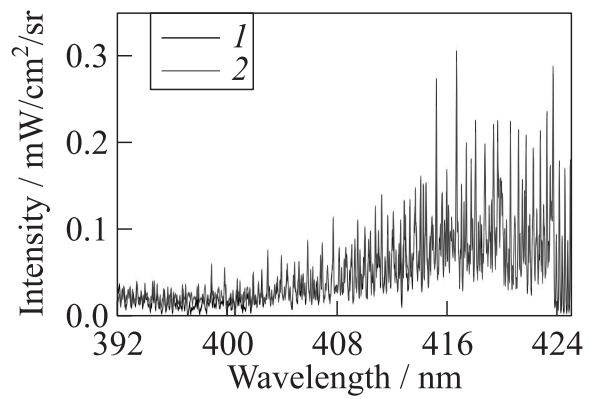

(a)

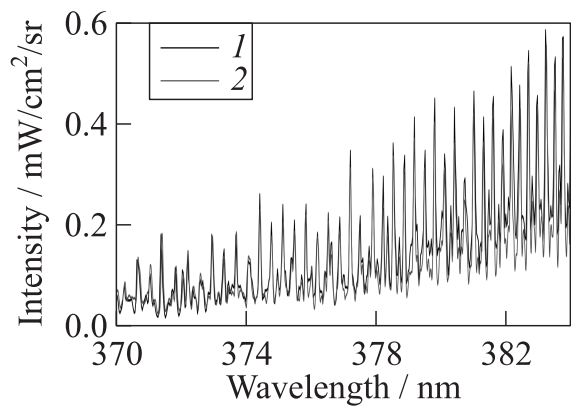

(c)

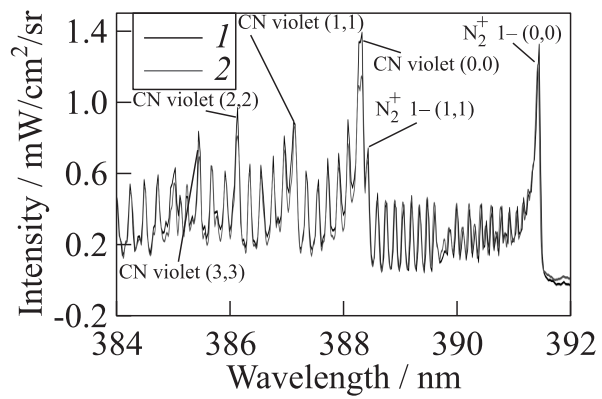

(b)

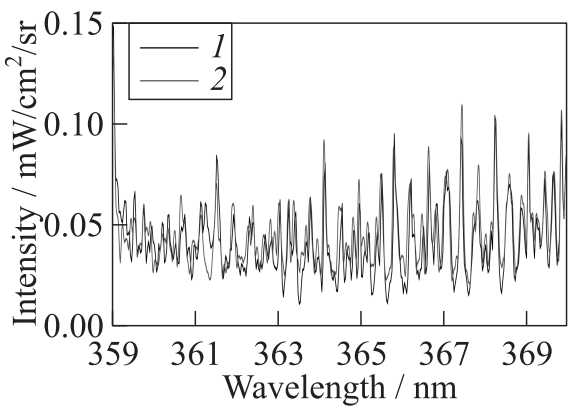

(d)

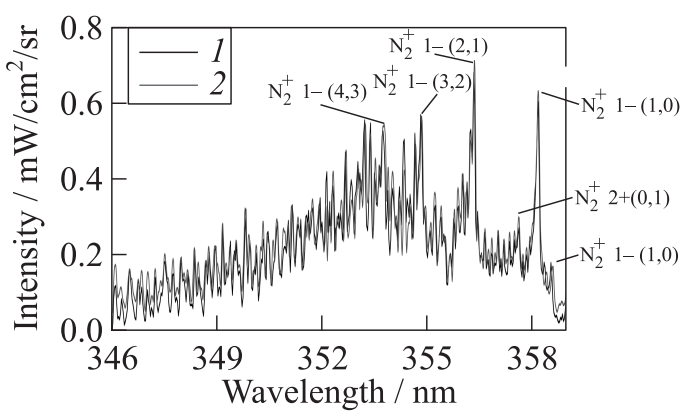

(e)

Figure 9 Measured with OL-550 (1) and SPECAIR (2) spectra between 392 and $425 \mathrm{~nm}(a) ; 384$ and $392 \mathrm{~nm}(b) ; 370$ and $384 \mathrm{~nm}(c) ; 359$ and $370 \mathrm{~nm}(d)$; and 346 and $360 \mathrm{~nm}(e)$

Between 392 and $425 \mathrm{~nm}$, SPECAIR closely reproduces the measured spectrum.

Between 384 and $392 \mathrm{~nm}$, as can be seen from Fig. 9b, SPECAIR closely reproduces the measured spectrum, in particular, the band systems of CN Violet and $\mathrm{N}_{2}^{+}(1-)$. It is also remarkable to note the attenuation of the intensity at 


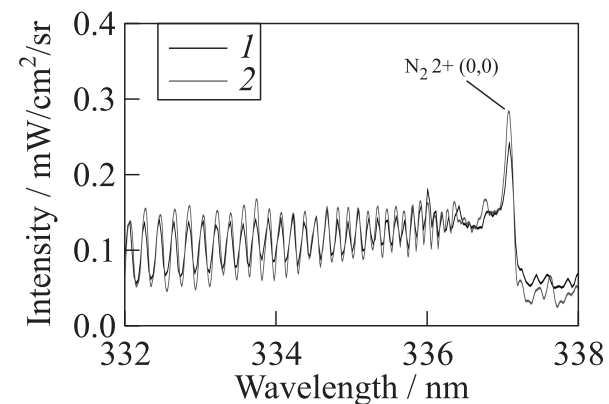

(a)

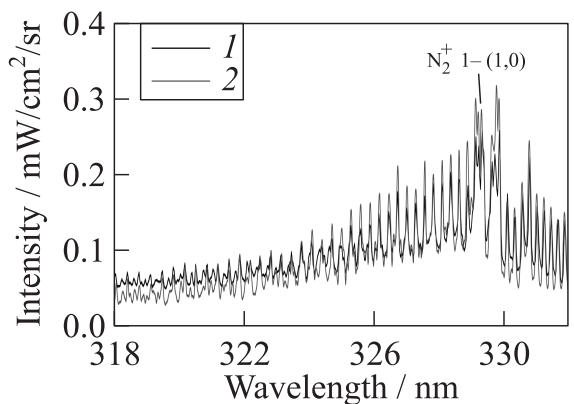

(b)

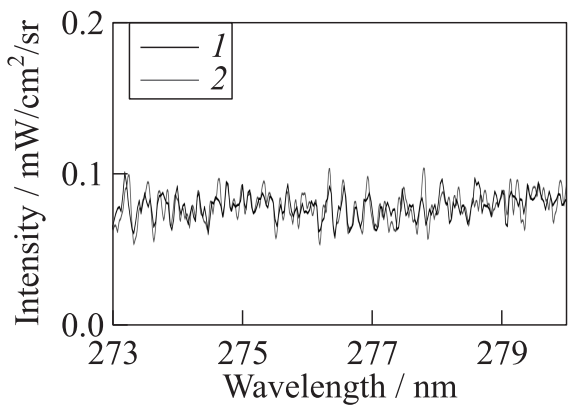

(c)

Figure 10 Measured (calibrated with the argon miniarc lamp) (1) and SPECAIR (2) spectra between 332 and $338 \mathrm{~nm}(a)$; between 318 and $332 \mathrm{~nm}(b)$; and between 273 and $280 \mathrm{~nm}(c)$

approximately $389.8 \mathrm{~nm}$, which is due to perturbations of the $\mathrm{N}_{2}^{+}(1-)$ transition, modeled in SPECAIR as described in [10] .

The measured and simulated spectra in the range 370-384 and 359-370 nm are shown in Figs. $9 c$ and $9 d$. This range corresponds to the emission of $\mathrm{N}_{2}^{+}$ $(1-)$, CN Violet, and $\mathrm{N}_{2}(2+)$. The agreement is again very good.

Between 346 and $360 \mathrm{~nm}$ (see Fig. $9 c$ ), the $\Delta v=1$ bands of $\mathrm{N}_{2}^{+}(1-), \mathrm{CN}$ Violet, and $\mathrm{N}_{2}(2+)$ are present in the measured spectrum. Every transition of the measured spectrum is explained by the SPECAIR calculation.

In Fig. $10 a$, the head of the $(0,0)$ band of $\mathrm{N}_{2}(2+)$ at $337 \mathrm{~nm}$ and a background of $\mathrm{NO} \beta$, NO $\gamma$, and $\mathrm{N}_{2}^{+}(1-)$ can be recognized from the measured spectrum between 332 and $338 \mathrm{~nm}$. Every transition in the measured spectrum is well predicted by SPECAIR.

Between 318 and $332 \mathrm{~nm}$ (see Fig. 10b) the measured spectrum includes $\mathrm{N}_{2}(2+)$ and $\mathrm{N}_{2}^{+}(1-)$ bands as well as a background of NO $\beta$ and $\gamma$. The experimental spectrum is again well reproduced by the simulations. 


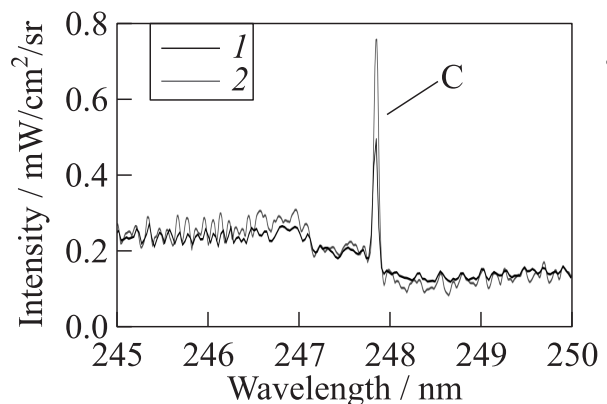

(a)

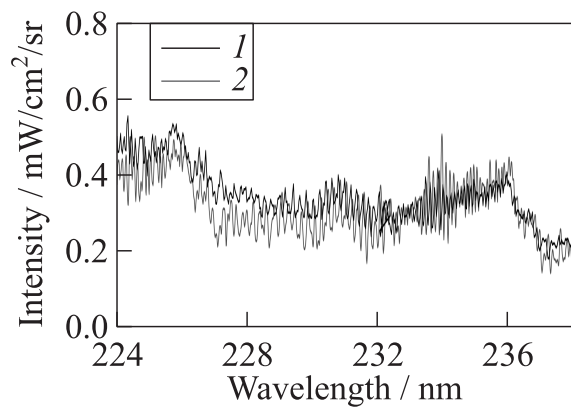

(c)

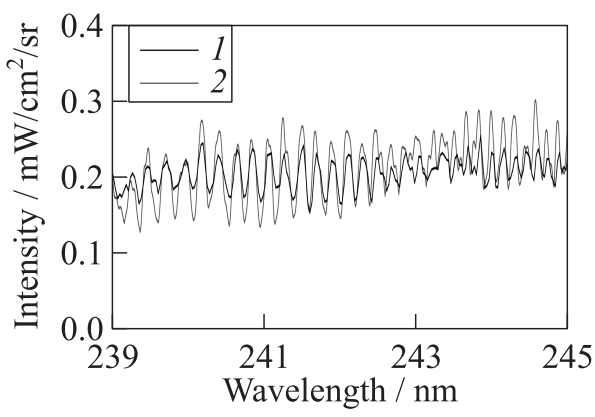

(b)

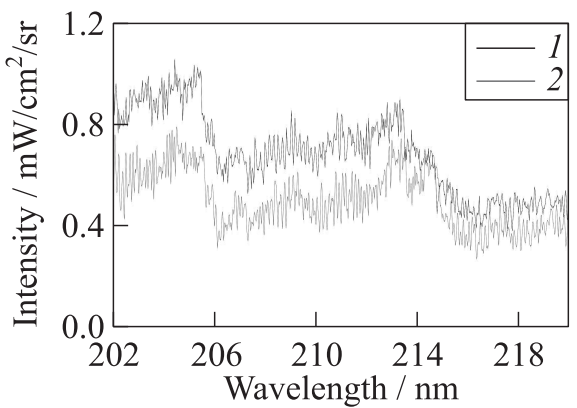

(d)

Figure 11 Measured (calibrated with the argon miniarc lamp) (1) and SPECAIR (2) spectra between 245 and $250 \mathrm{~nm}(a)$; between 239 and $245 \mathrm{~nm}(b)$; between 224 and $238 \mathrm{~nm}(c)$; and between 202 and $220 \mathrm{~nm}(d)$

Between 273 and $280 \mathrm{~nm}$ (see Fig. 10c), the spectrum mostly comprises the bands of $\mathrm{NO} \gamma, \mathrm{NO} \beta, \mathrm{NO} \delta, \mathrm{NO} \epsilon, \mathrm{O}_{2}$ Schumman-Range, and $\mathrm{N}_{2}(2+)$. Again good agreement is obtained, even though the signal-to-noise ratio is lower in this spectral range.

Figure $11 a$ shows spectra between 245 and $250 \mathrm{~nm}$, a range where the carbon line is present along with an underlying background of NO $\gamma$. A possible reason for the discrepancy could be a calibration uncertainty. However, it should also be noted that this difference may also be due to the uncertainty on the measured temperature (as mentioned earlier, a 3 percent change of the temperature causes a 60 percent change in the intensity of the carbon line).

Figure $11 b$ shows the spectrum from 239 to $245 \mathrm{~nm}$. Here, the spectrum is dominated by NO $\gamma$ emission bands, which appear to be well modeled.

From 224 to $238 \mathrm{~nm}$, seen in Fig. 11c, the two spectra are generally in good agreement. Here, the spectrum is dominated by NO $\gamma$ emission bands, with contributions of $\mathrm{NO} \beta, \delta$, and $\epsilon$ bands that start being significant. The range 


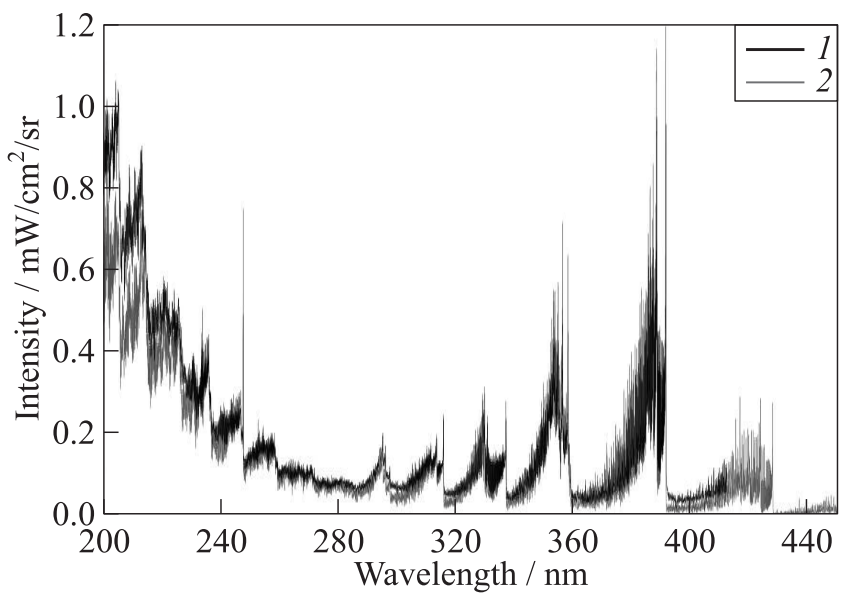

Figure 12 Comparison between the experimental (with argon miniarc) (1) and the SPECAIR calculated (2) spectra in absolute intensity between 200 and $400 \mathrm{~nm}$

between 224 and $227 \mathrm{~nm}$ shows some discrepancies that may be explained by the calibration as over this wavelength range, the argon miniarc does not emit strongly and the signal after background subtraction is very weak (only $22 \%$ of the measured signal, with the remaining $78 \%$ being straylight and electronic noise). This has a major influence on the spectrum. Further work could be done to improve calibration over this wavelength range.

In the last range, shown in Fig. $11 d$, the measured spectrum is about $50 \%$ higher than the simulation, but the overall shape is well reproduced. The difference may be from the calibration as explained earlier. Also, the highresolution spectrometer confirms the presence of either a potential missing additional species or uncertainties in the positions of some of the NO systems. Nevertheless, as can be seen by comparison with Fig. 13, the NO $\delta$ band system, in particular, appears to compare well with the experiments. The previously unexplained peak of SPECAIR at approximately $204.5 \mathrm{~nm}$ (Fig. 1) is now better predicted but there still appears to be a difference at about $214.5 \mathrm{~nm}$ These spectra thus provide valuable information to test future models of the strongly perturbed NO transitions in the range 200-230 nm.

Figure 13 shows the shape and the intensity of each NO emission band. This permits further analysis of the previous spectra. It shows which species emit where. It could be useful to identify species that might not be well modeled in order to best fit the experimental spectra and the SPECAIR one over the range $200-300 \mathrm{~nm}$. It is possible that some transitions are missing and, therefore, further analysis is needed to improve the knowledge over this wavelength range. 

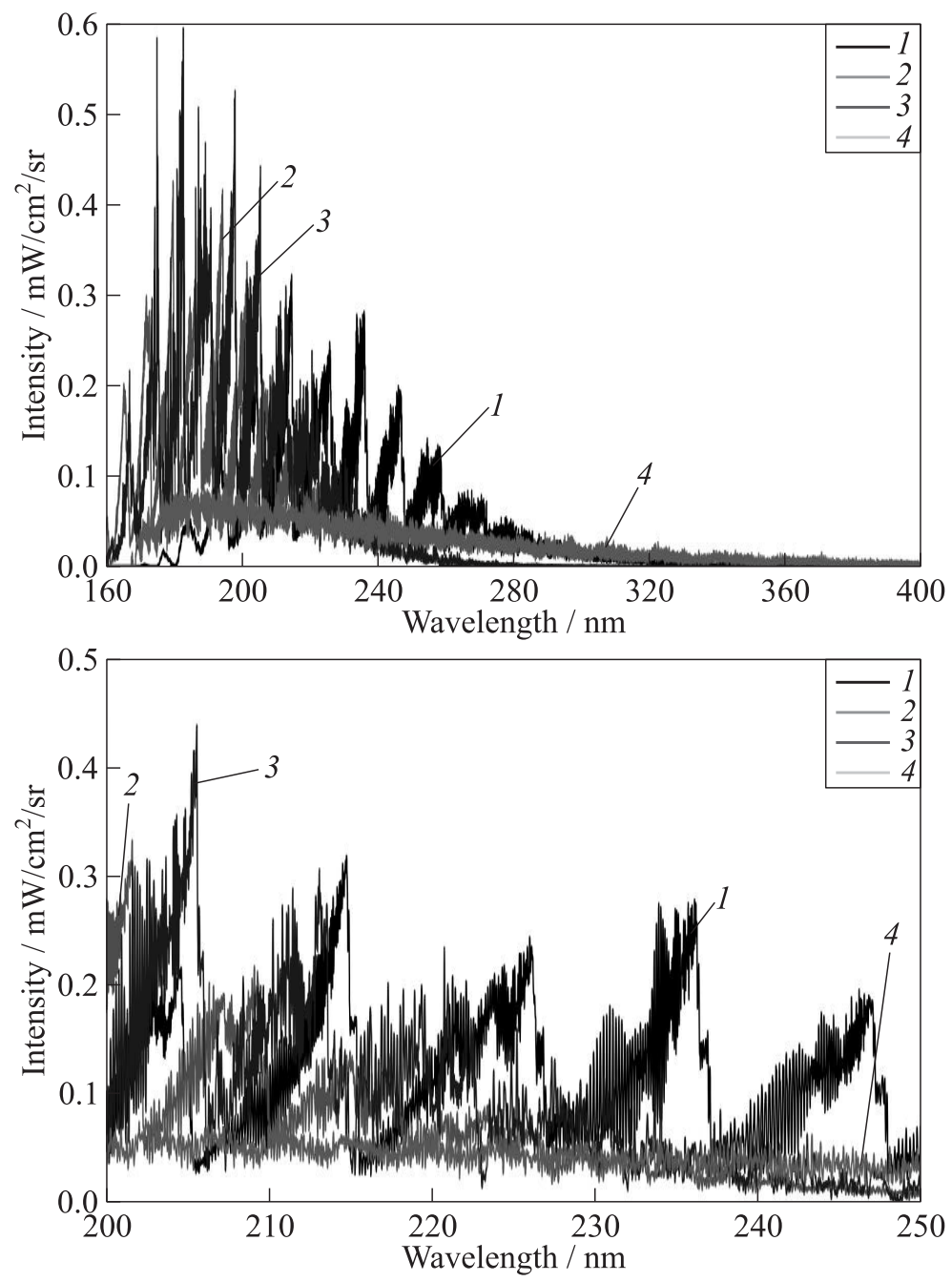

Figure 13 Contribution of different NO emission bands: $1-\gamma ; 2-\epsilon ; 3-\delta$; and $4-\beta$

\section{CONCLUDING REMARKS}

High-resolution emission spectra of an air plasma at a temperature of approximately $7500 \mathrm{~K}$ have been measured and calibrated in absolute intensity. These spectra were compared with simulations of the SPECAIR code. Most transitions observed in the experimental spectra are very well reproduced, both in shape and magnitude, by SPECAIR. In particular, the spectra of the $\mathrm{N}_{2}(2+)$, 
$\mathrm{N}_{2}^{+}(1-)$, and CN Violet agree very closely with the experiment. A few differences are noted over narrow spectral ranges such as $202-220 \mathrm{~nm}, 224-238 \mathrm{~nm}$, and $248 \mathrm{~nm}$. Differences are mainly in the range $200-220 \mathrm{~nm}$ where the measured spectra correspond to the superposition of at least six band systems of NO: $\beta$, $\gamma, \delta, \epsilon, \beta^{\prime}$, and $\gamma^{\prime}$ transitions. Some of these bands are strongly perturbed by interactions between the various electronic levels. The SPECAIR model takes into account these perturbations by incorporating the coupled $138 \times 138$ hamiltonian of the B, C, L, K, and Q stated of Gallusser and Dressler [13].

In the future, other models will be examined to improve spectral predictions, as some transitions might not be known and implemented in SPECAIR. The present measurements will provide a useful guide to test these models.

\section{REFERENCES}

1. Laux, C. O., T. G. Spence, C. H. Kruger, and R. N. Zare. 2003. Optical diagnostics of atmospheric pressure air plasmas. Plasma Sources Sci. Technol. 12:125-138. www.spectralfit.com.

2. Laux, C. O. 2007. Spectroscopic challenges in the modelling and diagnostics of high temperature air plasma radiation for aerospace applications. 5th Conference (International) on Atomic and Molecular Data and Their Applications.

3. Laux, C. O. 1993. Optical and radiative emission of high temperature air plasmas. Stanford University. Ph.D. Thesis.

4. Mac Donald, M. E., C. M., Jacobs, and C. O. Laux. 2013. Radiation shapes thermal protection investigations for high speed earth re-entry. ESA Report D4.2. Ablationradiation studies.

5. NASA RP-1311. 1994. Pt. I. 1996. Pt. II.

6. NIST. 2013. ASD Database.

7. Laux, C. O. 2008. Optical diagnostics and collisional-radiative models. VKI Course on hypersonic entry and cruise vehicles. Stanford University.

8. Packan, D. M., C. O. Laux, R. J. Gessman, L. Pierrot, and C. H. Kruger. 2003. Measurement and modeling of $\mathrm{OH}, \mathrm{NO}$, and $\mathrm{CO}_{2}$ infrared radiation. J. Thermophys. Heat Transfer 17(4):450-456.

9. Michaud, F., F. Roux, S.P. Davis, A.-D. Nguyen, and C. O. Laux. 2000. Highresolution fourier spectrometry of the $\mathrm{N}_{2}^{+}$ion. J. Molec. Spectrosc. 203:1-8.

10. Laux, C. O., R. J. Gessman, C. H. Kruger, F. Roux, F. Michaud, and S. P. Davis. 2001. Rotational temperature measurements in air and nitrogen plasmas using the first negative system of $\mathrm{N}_{2}^{+}$. J. Quant. Spectrosc. Radiative Transfer 68(4):473-482.

11. Levin, D. A., C. O. Laux, and C. H. Kruger. 1991. A general model for the spectral calculation of $\mathrm{OH}$ radiation in the ultraviolet. J. Quantitative Spectrosc. Radiative Transfer 61(3):377-392.

12. Caillault, L., J. Andreasson, J. Risberg, and C. O. Laux. 2006. Modeling the spectral radiation of a methane/nitrogen plasma. Test Case 4. 2nd Workshop (International) on Radiation of High Temperature Gases in Atmospheric Entry. Rome, Italy.

13. Gallusser, R., and K. Dressler. 1982. Multistate vibronic coupling between the excited ${ }^{2} \Pi$ stated of the NO molecule. J. Chem. Phys. 79:4311-4327. 